\title{
Body mass index and basal androstenedione are independent risk factors for miscarriage in polycystic ovary syndrome
}

Wan Yang ${ }^{1}$, Rui Yang ${ }^{1}$, Mingmei Lin ${ }^{1}$, Yan Yang ${ }^{1}$, Xueling Song ${ }^{1}$, Jiajia Zhang ${ }^{1}$, Shuo Yang ${ }^{1}$, Ying Song ${ }^{1}$, Jia Li ${ }^{1}$, Tianshu Pang ${ }^{1}$, Feng Deng ${ }^{1}$, Hua Zhang ${ }^{2}$, Ying Wang ${ }^{1 *}$, Rong $\mathrm{Li}^{1}$ and Jie Jiao ${ }^{1}$

\begin{abstract}
Background: There is limited literature investigating the effects of body mass index (BMI) and androgen level on in vitro fertilization (IVF) outcomes with a gonadotropin-releasing hormone (GnRH)-antagonist protocol in polycystic ovary syndrome (PCOS). Androgen-related variation in the effect of body mass index (BMI) on IVF outcomes remains unknown.

Methods: In this retrospective study, 583 infertile women with PCOS who underwent IVF using the conventional GnRH-antagonist protocol were included. Patients were divided into four groups according to BMI and androgen level: overweight- hyperandrogenism(HA) group, $n=96$, overweight-non-HA group, $n=117$, non-overweight-HA group, $n=152$, and non-overweight-non-HA group, $n=218$.

Results: A significantly higher number of oocytes were retrieved, and the total Gn consumption as well Gn consumption per day was significantly lower, in the non-overweight groups than in the overweight groups. The number of available embryos was significantly higher in the HA groups than in the non-HA groups. Clinical pregnancy rate was of no significant difference among four groups. Live-birth rates in the overweight groups were significantly lower than those in non-overweight-non-HA group $(23.9,28.4 \%$ vs. $42.5 \%, P<0.05)$. The miscarriage rate in overweight-HA group was significantly higher than that in non-overweight-non-HA group (45.2\% vs. $14.5 \%$, $P<0.05)$. Multivariate logistic regression analysis revealed that BMI and basal androstenedione (AND) both acted as significantly influent factors on miscarriage rate. The area under the curve (AUC) in receiver operating characteristic (ROC) analysis for $\mathrm{BMl}$ and basal AND on miscarriage rate were $0.607(P=0.029)$ and $0.657(P=0.001)$, respectively, and the cut-off values of BMl and basal AND were $25.335 \mathrm{~kg} / \mathrm{m}^{2}$ and $10.95 \mathrm{nmol} / \mathrm{L}$, respectively.

Conclusions: In IVF cycles with GnRH-antagonist protocol, economic benefits were seen in non-overweight patients with PCOS, with less Gn cost and more retrieved oocytes. BMI and basal AND were both significantly influential factors with moderate predictive ability on the miscarriage rate. The predictive value of basal AND on miscarriage was slightly stronger than BMI.
\end{abstract}

Keywords: Gonadotropin-releasing hormone antagonist, Polycystic ovary syndrome, Hyperandrogenism, Body mass index, In vitro fertilization

\footnotetext{
*Correspondence: wang_163ying@163.com

${ }^{1}$ Center for Reproductive Medicine, Department of Obstetrics and

Gynecology, Peking University Third Hospital, 49 N Garden Rd, Haidian

District, Beijing 100191, China

Full list of author information is available at the end of the article
}

(c) The Author(s). 2018 Open Access This article is distributed under the terms of the Creative Commons Attribution 4.0 International License (http://creativecommons.org/licenses/by/4.0/), which permits unrestricted use, distribution, and reproduction in any medium, provided you give appropriate credit to the original author(s) and the source, provide a link to the Creative Commons license, and indicate if changes were made. The Creative Commons Public Domain Dedication waiver (http://creativecommons.org/publicdomain/zero/1.0/) applies to the data made available in this article, unless otherwise stated. 


\section{Introduction}

For infertile women with polycystic ovary syndrome (PCOS) who fail lifestyle intervention and ovulation induction therapy or who have additional infertility factors, in vitro fertilization (IVF) can be used. Moderate evidence suggests that a gonadotropin (Gn)-releasing hormone $(\mathrm{GnRH})$-antagonist protocol can significantly reduce the incidence of ovarian hyperstimulation syndrome (OHSS) [1-3], and the use of a GnRH-antagonist protocol is gradually being adopted by clinicians. Phenotypic expressions of PCOS include oligo-ovulation/anovulation, hyperandrogenism (HA), polycystic ovaries, overweight/ obesity, and insulin resistance/metabolic syndrome [4]. There is limited literature investigating the clinical phenotype of patients with PCOS who can benefit the most from a $\mathrm{GnRH}$-antagonist protocol during IVF.

In China, $34.63 \%$ of patients with PCOS have a body mass index (BMI) above $23 \mathrm{~kg} / \mathrm{m}^{2}$ [5]. There are different opinions about the role of BMI in IVF outcomes. In 2016, Provost et al. [6] analyzed a total of 239,127 fresh IVF cycles and demonstrated a progressive and statistically significant worsening of outcomes in groups with higher BMIs, including cycles performed specifically for PCOS or male-factor infertility. Bailey et al. [7] also indicated that obese women with PCOS had lower odds of clinical pregnancy and live birth than lean women with PCOS, while there was a trend toward decreased OHSS incidence with increasing BMI among women with PCOS. However, Dechaud et al. [8] showed that obese patients required a higher recombinant follicle-stimulating hormone ( $\mathrm{r}-\mathrm{FSH})$ dose, but it did not have any adverse impact on IVF, including the cancellation rate, implantation rate, and pregnancy rate. Metwally et al. [9] found that obesity adversely affected embryo quality in young women undergoing IVF/intracytoplasmic sperm injection, while the oocyte quality was not affected.

The prevalence of biochemical HA in patients with PCOS is $78.2 \%$ [10]. Studies on the role of HA in IVF outcomes are limited. A recent study reported that HA in women with PCOS did not compromise IVF results, in contrast, facilitated the retrieval of a significantly higher number of oocytes [11]. In our recent study, HA also had a positive effect on the number of retrieved oocytes, where it is associated with miscarriage rate as well [12]. Conversely, another study inferred that the combination of HA and chronic anovulation was associated with a negative impact on the clinical pregnancy rate in patients with PCOS [13]. Endocrine disturbances are complicated in patients with PCOS. HA and obesity interact with each other and promote the progression of PCOS. However, few studies to date have investigated BMI and androgen level together. Therefore, the present study was designed to evaluate whether the effect of BMI on IVF outcomes vary with the level of androgen in PCOS with a GnRH-antagonist protocol.

\section{Materials and methods}

Between January 2013 and December 2015, a total of 583 infertile patients with PCOS treated with the conventional GnRH-antagonist protocol at the Center for Reproductive Medicine of Peking University Third Hospital were screened and enrolled in the study. Patients with PCOS between 20 and 35 years of age who were undergoing their first IVF cycle were included. Exclusion criteria included endometriosis, previous ovarian surgical history, hydrosalpinx, severe oligoasthenospermia or azoospermia, and systemic diseases such as diabetes mellitus and hypo- or hyperthyroidism. This retrospective cohort study was approved by the Institutional Review Board of the Department of Obstetrics and Gynecology, Peking University Third Hospital of Beijing, China.

In all cases, PCOS was diagnosed according to the Rotterdam 2003 criteria [14]. BMI was calculated by the following formula: $\mathrm{BMI}=$ weight $/$ height $^{2}\left(\mathrm{~kg} / \mathrm{m}^{2}\right)$. HA was diagnosed by a testosterone level $\geq 2.2 \mathrm{nmol} / \mathrm{L}$ or AND level $\geq 12 \mathrm{nmol} / \mathrm{L}$. Testosterone and AND levels were obtained from basal sex hormone assessments. This cohort study included four groups of patients with PCOS: BMI $\geq 25 \mathrm{~kg} / \mathrm{m}^{2}$ with HA (overweight-HA group, $n=96$ ), BMI $\geq 25 \mathrm{~kg} / \mathrm{m}^{2}$ with normal androgen (overweight-non-HA group, $n=117$ ), BMI $<25 \mathrm{~kg} / \mathrm{m}^{2}$ with HA (non-overweight-HA group, $n=152$ ), and $\mathrm{BMI}<25 \mathrm{~kg} / \mathrm{m}^{2}$ with normal androgen (non-overweight-non-HA group, $n=218$ ).

All patients were stimulated with a combination of a GnRH antagonist (cetrorelix; SeronoMunich, Germany) and an r-FSH drug (Gonal-f; Merck Serono, Munich, Germany) to develop multiple follicles. Controlled ovarian hyperstimulation was started from 2nd day of the menstrual period when no follicle $>10 \mathrm{~mm}$ in diameter was detected by ultrasound, and serum estradiol levels were $<50 \mathrm{pg} / \mathrm{mL}$. The $\mathrm{Gn}$ dose was modified in accordance with the ovarian response. Highly purified human menopausal gonadotropin (menogon; Ferring, Kiel, Germany) was occasionally added. A GnRH antagonist was injected subcutaneously at a daily dose of $0.25 \mathrm{mg}$ when follicles with a mean diameter $\geq 14 \mathrm{~mm}$ were detected. The criterion for administration of recombinant human chorionic gonadotropin $(\mathrm{hCG})(250 \mu \mathrm{g})$ was the observation of at least two follicles with a mean diameter $\geq 18 \mathrm{~mm}$. Thirty-six hours after the trigger, oocyte retrieval was performed with ultrasound guidance, using a 16-G double-lumen aspiration needle. Conventional fertilization and embryo culture were performed. In cases where the number of retrieved oocytes was $\geq 20$ or estradiol levels were above $15,000 \mathrm{pmol} / \mathrm{L}$ on the day of hCG administration, all available embryos were cryopreserved by the vitrification method for future transfer to prevent OHSS. Two embryos were transferred three days after oocyte retrieval. Supportive therapy with progesterone 
was administered vaginally ( $90 \mathrm{mg}$ daily), starting on the day of oocyte collection, and was continued until 10 weeks of gestation.

Primary outcome measures were rate of clinical pregnancy, miscarriage rate, and rate of live birth. Secondary outcome measures were Gn dosage, number of oocytes collected, and number of available embryos.

Data were analyzed with SPSS 20.0 (Chicago, IL). The chi-square test was used for categorical variables, and analysis of variance was used for continuous variables. Logistic regression analysis was performed using these binary variables in a forward stepwise method. The optimal cut-off values were calculated by receiver operating characteristic (ROC) analysis using the Youden index. A $P$ value of $<0.05$ was considered statistically significant for all analyses.

\section{Results}

Baseline characteristics of patients are shown in Table 1. There were no significant differences in age, basal FSH, and antral follicle count between the groups. The duration of infertility was significantly higher in the overweight groups than in the non-overweight groups. The basal luteinizing hormone (LH) levels were significantly higher in the HA groups than in the non-HA groups.

Characteristics of ovarian responses among the four patient groups are shown in Table 2. The number of oocytes retrieved and the estradiol levels on the day of hCG administration were significantly higher in the non-overweight groups than in the overweight groups, although the total Gn consumption, as well as Gn consumption per day, was significantly lower in the non-overweight groups. Despite the effect of BMI, Gn consumption per kilogram was significantly lower in the HA groups. As with the number of oocytes retrieved, the cancellation rates of embryo transfer to prevent OHSS were significantly higher in the non-overweight groups than in the overweight groups. Unlike the basal LH levels that were related to basal androgen levels, LH levels on the day of hCG administration were significantly lower in the non-overweight groups than in the overweight groups. The number of available embryos was higher in non-overweight-HA group than in overweight-non-HA group. The level of progesterone (P4) and the endometrial thickness on the day of hCG administration were not different among the four groups. Similarly, the fertilization rate and the cleavage rate were comparable among the four groups.

Pregnancy outcomes are presented in Table 3. The miscarriage rate was significantly higher in overweightHA group than in non-overweight-non-HA group. The live-birth rates were significantly lower in the overweight groups than in non-overweight-non-HA group. There were no ectopic pregnancies in HA groups. There were no differences in the rates of clinical pregnancy, preterm pregnancy, twin pregnancy, or caesarean section among the study groups.

Multivariate logistic regression analyses and ROC analysis were performed to define the predictive factors of miscarriage rate. Parameters that could have adverse effects on miscarriage rate were age, BMI, basal testosterone, basal AND, Gn consumption $/ \mathrm{kg} /$ day, and number of oocytes retrieved. These parameters, as well as LH levels (both basal LH and LH on the day of hCG administration), were chosen for multivariate logistic regression analyses. After adjusting the effects of other parameters, BMI and basal AND showed independently significant differences in predicting the miscarriage rate (Table 4). A ROC analysis was performed to define the optimal cut-off values of BMI and basal AND (Fig. 1). The areas under the curve (AUCs) for the BMI and basal AND were $0.607(P=0.029)$ and $0.657(P=0.001)$, respectively (Table 5$)$. These two parameters were demonstrated to have moderate predictive ability on miscarriage, with a sensitivity of $0.511-0.723$ and a specificity of $0.584-0.737$.

Table 1 Baseline characteristics of patients

\begin{tabular}{|c|c|c|c|c|c|}
\hline \multirow[t]{2}{*}{ Parameter } & \multicolumn{2}{|c|}{ Non-overweight } & \multicolumn{2}{|l|}{ Overweight } & \multirow[t]{2}{*}{$P$ value } \\
\hline & $\begin{array}{l}\text { Non-HA } \\
(n=218)\end{array}$ & $\begin{array}{l}\mathrm{HA} \\
(n=152)\end{array}$ & $\begin{array}{l}\text { Non-HA } \\
(n=117)\end{array}$ & $\begin{array}{l}\mathrm{HA} \\
(n=96)\end{array}$ & \\
\hline Age (y) & $29.2 \pm 3.6$ & $29.0 \pm 3.4$ & $29.8 \pm 3.2$ & $29.1 \pm 3.2$ & NS \\
\hline $\mathrm{BMI}\left(\mathrm{kg} / \mathrm{m}^{2}\right)$ & $21.5 \pm 2.1^{\mathrm{a}}$ & $21.6 \pm 2.2^{a}$ & $28.5 \pm 2.3^{b}$ & $28.7 \pm 3.1^{b}$ & $<0.001$ \\
\hline Infertile duration (y) & $3.7 \pm 2.5^{\mathrm{a}}$ & $3.7 \pm 2.1^{\mathrm{a}}$ & $4.6 \pm 2.7^{b}$ & $4.4 \pm 2.8^{b}$ & $<0.001$ \\
\hline Basal FSH (IU/L) & $6.1 \pm 1.5$ & $6.3 \pm 1.8$ & $5.9 \pm 1.8$ & $6.1 \pm 1.7$ & NS \\
\hline Basal LH (IU/L) & $5.7 \pm 3.8^{a}$ & $9.7 \pm 5.9^{b}$ & $5.5 \pm 3.8^{\mathrm{a}}$ & $8.9 \pm 4.6^{b}$ & $<0.001$ \\
\hline Basal T(ng/ml) & $0.8 \pm 0.3^{a}$ & $1.4 \pm 1.0^{b}$ & $0.9 \pm 0.3^{a}$ & $2.0 \pm 3.0^{c}$ & $<0.001$ \\
\hline Basal AND (nmol/L) & $8.0 \pm 2.5^{\mathrm{a}}$ & $17.3 \pm 6.0^{b}$ & $8.6 \pm 2.3^{a}$ & $19.2 \pm 7.3^{c}$ & $<0.001$ \\
\hline Total AFC (n) & $19.0 \pm 6$ & $19.8 \pm 5$ & $19.1 \pm 6$ & $20.8 \pm 7$ & NS \\
\hline
\end{tabular}

Data are presented as mean \pm standard deviation unless otherwise specified

NS not significant; HA hyperandrogenism; $A F C$ antral follicle count; BMI body mass index; $F S H$ follicle-stimulating hormone; $L H$ luteinizing hormone; $T$ testosterone; AND androstenedione

${ }^{a}$ Significantly different from ${ }^{b}$ or ${ }^{c}$ groups 
Table 2 Characteristics of ovarian responses

\begin{tabular}{|c|c|c|c|c|c|}
\hline \multirow[t]{2}{*}{ Parameter } & \multicolumn{2}{|l|}{ Non-overweight } & \multicolumn{2}{|l|}{ Overweight } & \multirow{2}{*}{$\begin{array}{l}P \\
\text { value }\end{array}$} \\
\hline & $\begin{array}{l}\text { Non-HA } \\
(n=218)\end{array}$ & $\begin{array}{l}\mathrm{HA} \\
(n=152)\end{array}$ & $\begin{array}{l}\text { Non-HA } \\
(n=117)\end{array}$ & $\begin{array}{l}\mathrm{HA} \\
(n=96)\end{array}$ & \\
\hline Gn stimulation days & $10.5 \pm 2.1^{a}$ & $9.8 \pm 1.8^{b}$ & $11.3 \pm 2.6^{c}$ & $10.5 \pm 2.4^{a}$ & $<0.001$ \\
\hline Total Gn dosage (IU) & $1588 \pm 659^{a}$ & $1420 \pm 595^{\mathrm{a}}$ & $2081 \pm 885^{b}$ & $1828 \pm 750^{c}$ & $<0.001$ \\
\hline Gn dosage /day (IU) & $148 \pm 40^{\mathrm{a}}$ & $141 \pm 35^{\mathrm{a}}$ & $180 \pm 49^{b}$ & $170 \pm 40^{c}$ & $<0.001$ \\
\hline Gn consumption/kg (IU) & $28.3 \pm 12^{\mathrm{a}}$ & $25.1 \pm 10^{b}$ & $27.6 \pm 11$ & $24.9 \pm 10^{b}$ & $<0.05$ \\
\hline Gn consumption/ kg/day & $2.6 \pm 0.7^{a}$ & $2.5 \pm 0.6$ & $2.4 \pm 0.6$ & $2.3 \pm 0.5^{b}$ & $<0.001$ \\
\hline E2 on HCG day (pmol/L) & $12,708 \pm 7621^{a}$ & $13,663 \pm 8165^{a}$ & $9219 \pm 6685^{b}$ & $11,143 \pm 7556$ & $<0.001$ \\
\hline LH on HCG day $(\mathrm{mlU} / \mathrm{mL})$ & $2.4 \pm 2.2^{\mathrm{a}}$ & $2.4 \pm 2.3^{\mathrm{a}}$ & $3.0 \pm 3.6$ & $3.2 \pm 2.6^{b}$ & $<0.001$ \\
\hline P4 on HCG day (nmol/L) & $2.4 \pm 1.4$ & $2.4 \pm 1.4$ & $2.2 \pm 1.3$ & $2.5 \pm 1.5$ & NS \\
\hline Endometrial thickness & $10.7 \pm 1.7$ & $10.4 \pm 1.7$ & $10.7 \pm 1.8$ & $10.2 \pm 1.9$ & NS \\
\hline Oocyte retrieved (n) & $16.7 \pm 8.4^{\mathrm{a}}$ & $18.2 \pm 9.9^{a}$ & $12.1 \pm 6.9^{b}$ & $13.8 \pm 8.5^{b}$ & $<0.001$ \\
\hline ET cancellation rate ${ }^{d}$ & $26.6 \%^{\mathrm{a}}$ & $35.5 \%^{\mathrm{a}}$ & $14.5 \%^{\mathrm{b}}$ & $18.8 \%^{b}$ & $<0.05$ \\
\hline Fertilization rate (\%) & $78.0 \pm 19$ & $76.5 \pm 19$ & $76.2 \pm 24$ & $75.1 \pm 21$ & NS \\
\hline Cleavage rate (\%) & $96.7 \pm 11$ & $98.9 \pm 03$ & $98.6 \pm 05$ & $97.6 \pm 08$ & NS \\
\hline Available embryos & $7.75 \pm 5.6$ & $8.91 \pm 6.9^{a}$ & $5.34 \pm 4.7^{b}$ & $6.68 \pm 6.0$ & $<0.001$ \\
\hline
\end{tabular}

Data are presented as mean \pm standard deviation unless otherwise specified

NS not significant; $B M I$ body mass index; HA hyperandrogenemia; PCOS polycystic ovary syndrome; Gn gonadotropin; E2 estradiol; $L H$ luteinizing hormone; $P 4$ progesterone; $h C G$ human chorionic gonadotropin; $E T$ embryo transfer

${ }^{\text {a }}$ Significantly different from ${ }^{\mathrm{b}}$ or ${ }^{\mathrm{c}}$ groups

${ }^{\mathrm{d}}$ The cancellation rate of embryo transfer to prevent ovarian hyperstimulation syndrome

Cut-off values were as follows: BMI, $25.335 \mathrm{~kg} / \mathrm{m}^{2}$; and basal AND, $10.95 \mathrm{nmol} / \mathrm{L}$.

\section{Discussion}

Nearly $70-80 \%$ of anovulatory infertility cases are caused by PCOS [15]. As a therapy, IVF can be safely used in infertile women with PCOS, especially with a $\mathrm{GnRH}$-antagonist protocol. There is few studies to date have investigated the parameters of BMI and androgen together with the use of GnRH antagonists. In this study, the aim was to assess Androgen-related variation in the effect of body mass index (BMI) on IVF outcomes.
In this study, the duration of infertility was significantly higher in the overweight groups than in the non-overweight groups, indicating that body weight may contribute to a longer duration of infertility. The number of oocytes retrieved was significantly higher in the non-overweight groups than in the overweight groups, although the total Gn consumption as well as Gn consumption per day was significantly lower. There is an economic benefit of using a $\mathrm{GnRH}$-antagonist protocol in non-overweight patients with PCOS, because of less cost and larger number of retrieved oocytes. Despite the effect of BMI, Gn consumption per kilogram was significantly

Table 3 Pregnancy outcomes

\begin{tabular}{|c|c|c|c|c|c|}
\hline \multirow[t]{2}{*}{ Parameter } & \multicolumn{2}{|l|}{ Non-overweight } & \multicolumn{2}{|l|}{ Overweight } & \multirow{2}{*}{$\begin{array}{l}P \\
\text { value }\end{array}$} \\
\hline & $\begin{array}{l}\text { Non-HA } \\
(n=218)\end{array}$ & $\begin{array}{l}\mathrm{HA} \\
(n=152)\end{array}$ & $\begin{array}{l}\text { Non-HA } \\
(n=117)\end{array}$ & $\begin{array}{l}\mathrm{HA} \\
(n=96)\end{array}$ & \\
\hline Clinical PR & $49.7 \%(76 / 153)$ & $43.6 \%(41 / 94)$ & $40.9 \%(36 / 88)$ & $43.7 \%(31 / 71)$ & NS \\
\hline Miscarriage rate & $14.5 \%(11 / 76)^{a}$ & $26.8 \%(11 / 41)$ & $30.6 \%(11 / 36)$ & $45.2 \%(14 / 31)^{\mathrm{b}}$ & $<0.05$ \\
\hline Live-birth rate & $42.5 \%(65 / 153)^{\mathrm{a}}$ & $31.9 \%(30 / 94)$ & $28.4 \%(25 / 88)^{b}$ & $23.9 \%(17 / 71)^{\mathrm{b}}$ & $<0.05$ \\
\hline Ectopic PR & $3.3 \%(5 / 153)$ & $0 \%(0 / 94)^{a}$ & $5.7 \%(5 / 88)^{b}$ & $0 \%(0 / 71)$ & $<0.05$ \\
\hline Singleton PR & $60.5 \%(46 / 76)$ & $56.1 \%(23 / 41)$ & $58.3 \%(21 / 36)$ & $58.1 \%(18 / 31)$ & NS \\
\hline Twin PR & $39.5 \%(30 / 76)$ & $43.9 \%(18 / 41)$ & $41.7 \%(15 / 36)$ & $41.9 \%(13 / 31)$ & NS \\
\hline Preterm rate & $22.4 \%(17 / 76)$ & $19.5 \%(8 / 41)$ & $19.4 \%(7 / 36)$ & $12.9 \%(4 / 31)$ & NS \\
\hline Full term rate & $63.2 \%(48 / 76)$ & $53.7 \%(22 / 41)$ & $50.0 \%(18 / 36)$ & $41.9 \%(13 / 31)$ & NS \\
\hline CS rate & $72.3 \%(47 / 65)$ & $70.0 \%(21 / 30)$ & $84.0 \%(21 / 25)$ & $94.1 \%(16 / 17)$ & NS \\
\hline
\end{tabular}

HA hyperandrogenemia; PCOS polycystic ovary syndrome; $B M I$ body mass index; NS not significant; $P R$ pregnancy rate; $C S$ caesarean section

a Significantly different from ${ }^{\mathrm{b}}$ groups 
Table 4 Multivariate logistic regression analysis for predictive factors of miscarriage rate

\begin{tabular}{lllll}
\hline Parameter & $\beta$ & $P$ value & Odds ratio & $\mathrm{Cl}(95 \%)$ \\
\hline Age $(\mathrm{y})$ & 0.040 & 0.502 & 1.040 & $0.927-1.168$ \\
BMl $\left(\mathrm{kg} / \mathrm{m}^{2}\right)^{\mathrm{a}}$ & 0.093 & 0.049 & 1.097 & $1.000-1.204$ \\
Basal LH (IU/L) & 0.030 & 0.379 & 1.031 & $0.963-1.103$ \\
Basal T(ng/ml) & 0.095 & 0.684 & 1.100 & $0.695-1.742$ \\
Basal AND $(\mathrm{nmol} / \mathrm{L})^{\mathrm{a}}$ & 0.069 & 0.034 & 1.071 & $1.005-1.141$ \\
Gn consumption/kg/day & 0.579 & 0.061 & 1.785 & $0.974-3.273$ \\
LH on HCG day $(\mathrm{m} / \mathrm{U} / \mathrm{mL})$ & 0.061 & 0.258 & 1.063 & $0.957-1.180$ \\
Oocyte retrieved $(\mathrm{n})$ & 0.022 & 0.533 & 1.023 & $0.953-1.098$ \\
Constant & -7.630 & 0.001 & 0.000 & \\
\hline
\end{tabular}

$B M I$ body mass index; $L H$ luteinizing hormone; $T$ testosterone; $A N D$ androstenedione; $\mathrm{Cl}$ confidence interval; $\mathrm{Gn}$ gonadotropin; $h C G$ human chorionic gonadotropin

${ }^{\text {a }}$ Significant difference

lower in the HA groups, indicating that when overweight PCOS patients lose weight, patients with HA gain economic benefits. The number of available embryos was higher in non-overweight-HA group than in overweight-non-HA group, indicating that a non-overweight build and over-secretion of androgen may be protective factors for good-quality embryos. The miscarriage rate was significantly higher in overweight-HA group than in non-overweight-non-HA group, indicating that being overweight and having HA may play an important role in higher miscarriage rates. It appeared that being overweight was a risk factor for both low-quality embryos and miscarriage. However, HA was a positively influential
Table 5 ROC analysis of BMI and AND on prediction of miscarriage rate

\begin{tabular}{llllll}
\hline Parameter & Cut-off value & AUC & $P$ value & Sensitivity & Specificity \\
\hline BMl $\left(\mathrm{kg} / \mathrm{m}^{2}\right)$ & 25.335 & 0.607 & 0.029 & 0.511 & 0.737 \\
$\begin{array}{l}\text { Basal AND } \\
\text { (nmol/L) }\end{array}$ & 10.95 & 0.657 & 0.001 & 0.723 & 0.584 \\
\hline
\end{tabular}

$B M I$ body mass index; $A N D$ androstenedione; $R O C$ receiver operating characteristic; AUC area under the curve

factor for good-quality embryos but a risk factor for miscarriage. Meanwhile, in multivariate logistic regression analyses, BMI and basal AND showed independently significant differences on prediction of miscarriage, after considering the effects of other parameters that may cause miscarriage. ROC analysis showed that the optimal cut-off values of BMI and basal AND were $25.335 \mathrm{~kg} / \mathrm{m}^{2}$ and $10.95 \mathrm{nmol} / \mathrm{L}$, respectively. Although the predictive abilities of both BMI and basal AND on miscarriage were moderate, with a sensitivity of $0.511-0.723$ and a specificity of $0.584-0.737$, they may still provide predictive values in clinical treatment since the AUCs for BMI and basal AND level were significantly different $(P=0.029$ and $P=0.001$, respectively). In addition, the predictive value of basal AND on miscarriage was slightly stronger than that of BMI.

PCOS is closely related to miscarriages. The molecular mechanisms underlying PCOS-associated miscarriages are controversial. Obesity and HA are factors that may contribute to miscarriage [13], which was confirmed by our previous study. By observational analysis of the developmental kinetics and metabolic activity of oocytes,

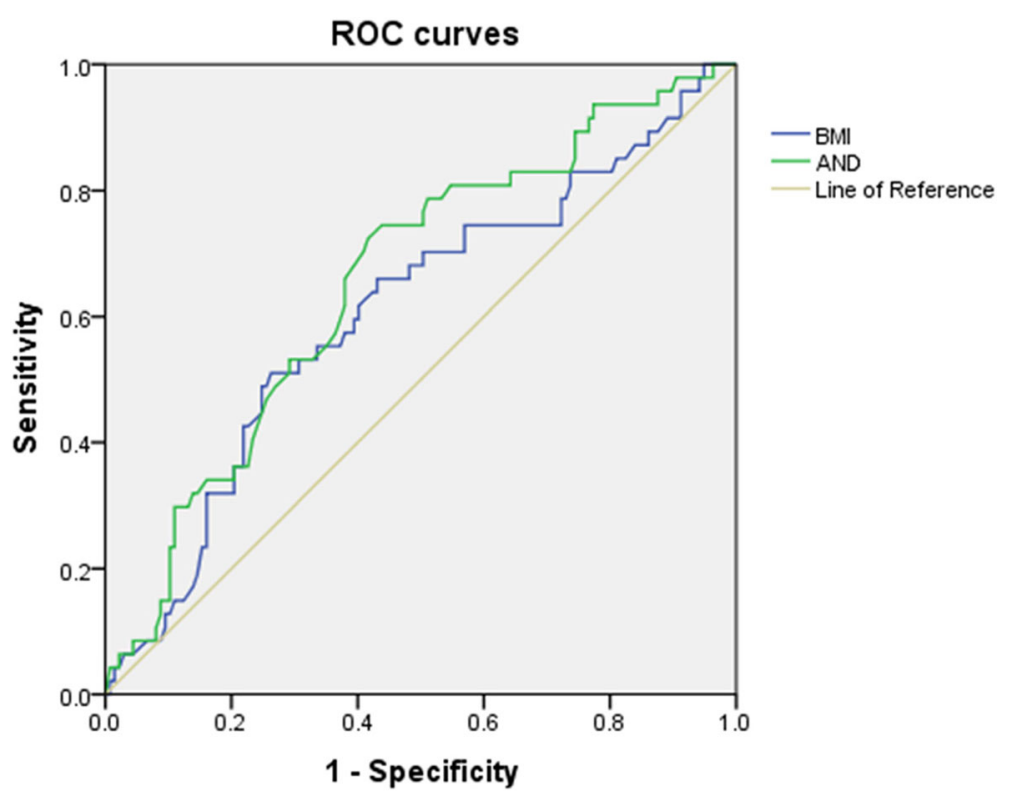

Fig. 1 Receiver operating characteristic (ROC) curves analysis of body mass index (BMI) and androstenedione (AND) on prediction of miscarriage rate. The green line and blue lines represent AND and BMI, respectively, and the beige line is the reference 
some researchers have observed that oocytes from women who are overweight or obese are smaller than those from women of healthy weight $\left(\mathrm{BMI}<25 \mathrm{~kg} / \mathrm{m}^{2}\right)$, yet post-fertilization they reach the morula stage faster and, as blastocysts, show reduced glucose consumption and elevated endogenous triglyceride levels [16]. The data indicate that a high BMI in women is associated with distinct phenotypic changes in the embryo, which may reduce the quality of embryos and cause miscarriage. Valckx et al. showed that metabolic alterations in the serum were reflected in the follicular fluid, and that some of these alterations may have affected oocyte quality; however, there were poor BMI-related associations [17]. As for HA-related miscarriages, there are several potential mechanisms. First, the basal LH levels are related to basal androgen levels, as shown in our study. HA contributes to the secretion of excessive amounts of LH that may cause oocyte maturation disturbances and miscarriage. Second, HA may have an adverse effect on ovarian folliculogenesis and granulosa cell function. Follicular atresia is potentiated by androgens in the immature rat, and granulose cell apoptosis in rats is inducible by androgens [18]. Increased expression of AKT1 and AKT2 may be a possible mechanism linking HA to granulosa cell dysfunction in patients with HA PCOS [19]. Finally, testosterone may impact the endometrium. Testosterone was reported to have a dose-dependent negative effect on the proliferation of decidualized endometrial stromal cells [20] and to suppress the expression of HOXA10, which is essential to endometrial receptivity [21]. In patients with PCOS, HA and obesity interact with each other and promote the progression of PCOS.

One limitation of our study is its retrospective design. The effects of BMI and HA are assessed in the cohort study with logistic regression analysis and ROC analysis, showing the optimal cut-off values of BMI and basal AND. Although AUCs in ROC analysis are significantly different, the predictive ability of both parameters on miscarriage is moderate, due to the relatively limited sample size. Prospective studies with a larger sample size are needed in the future, along with studies investigating the effects of hyperinsulinemia.

\section{Conclusions}

In conclusion, being overweight was a risk factor for both low-quality embryos and miscarriage. However, HA was a positively influential factor for good-quality embryos but a risk factor for miscarriage. Multivariate logistic regression analyses show that BMI and basal AND were both significantly influential factors on miscarriage rate, with moderate predictive ability. The predictive value of basal AND on miscarriage was slightly stronger than BMI.

\section{Acknowledgments}

The authors thank the Research Center of Clinical Epidemiology, Peking University Third Hospital, for support in statistical analyses. The authors thank Rozella, an experienced editor whose first language is English, for carefully reviewing the article.

\section{Funding}

This work was supported by the National Natural Science Foundation of China [81550022].

\section{Availability of data and materials}

The data sets used and/or analyzed during the current study are available from the database of Center for Reproductive Medicine in Peking University Third Hospital on reasonable request.

\section{Authors' contributions}

WY designed research. WY, RY, MML, SY, JJZ, YY, SLS, YS and TSP collected data. WY, FD, JL, HZ analyzed data. WY, YW, RL and JQ wrote and revised the manuscript. All authors read and approved the final manuscript.

\section{Ethics approval and consent to participate}

This study was approved by the Institutional Review Board of the Department of Obstetrics and Gynecology, Peking University Third Hospital. Since this is a retrospective investigation, patients were not asked to participate in this study.

\section{Consent for publication}

Not applicable.

Competing interests

The authors declare that they have no competing interests.

\section{Publisher's Note}

Springer Nature remains neutral with regard to jurisdictional claims in published maps and institutional affiliations.

\section{Author details}

${ }^{1}$ Center for Reproductive Medicine, Department of Obstetrics and Gynecology, Peking University Third Hospital, 49 N Garden Rd, Haidian District, Beijing 100191, China. ${ }^{2}$ Research Center of Clinical Epidemiology, Peking University Third Hospital, Beijing, China.

Received: 15 August 2018 Accepted: 13 November 2018

Published online: 19 November 2018

\section{References}

1. Balen AH, Morley LC, Misso M, et al. The management of anovulatory infertility in women with polycystic ovary syndrome: an analysis of the evidence to support the development of global WHO guidance. Hum Reprod Update. 2016;22(6):687-708.

2. Kollmann M, Martins WP, Lima ML, et al. Strategies for improving outcome of assisted reproduction in women with polycystic ovary syndrome: systematic review and meta-analysis. Ultrasound Obstet Gynecol. 2016;48(6): 709-18.

3. Xiao J, Chen S, Zhang C, et al. Effectiveness of GnRH antagonist in the treatment of patients with polycystic ovary syndrome undergoing IVF: a systematic review and meta-analysis. Gynecol Endocrinol. 2013;29(3):187-91.

4. Fauser BC, Tarlatzis BC, Rebar RW, et al. Consensus on women's health aspects of polycystic ovary syndrome (PCOS): the Amsterdam ESHRE/ASRM-sponsored 3rd PCOS consensus workshop group. Fertil Steril. 2012;97(1):28-38.

5. Chen $\mathrm{X}, \mathrm{Ni} \mathrm{R}, \mathrm{Mo} \mathrm{Y}$, et al. Appropriate BMI levels for PCOS patients in southern China. Hum Reprod. 2010;25(5):1295-302.

6. Provost MP, Acharya KS, Acharya CR, et al. Pregnancy outcomes decline with increasing body mass index: analysis of 239,127 fresh autologous in vitro fertilization cycles from the 2008-2010 Society for Assisted Reproductive Technology registry. Fertil Steril. 2016;105(3):663-9.

7. Bailey AP, Hawkins LK, Missmer SA, et al. Effect of body mass index on in vitro fertilization outcomes in women with polycystic ovary syndrome. Am J Obstet Gynecol. 2014;211(2):163-e1. 
8. Dechaud $H$, Anahory T, Reyftmann $L$, et al. Obesity does not adversely affect results in patients who are undergoing in vitro fertilization and embryo transfer. Eur J Obstet Gynecol Reprod Biol. 2006;127(1):88-93.

9. Metwally $M$, Cutting $R$, Tipton $A$, et al. Effect of increased body mass index on oocyte and embryo quality in IVF patients. Reprod BioMed Online. 2007; 15(5):532-8.

10. Alexiou E, Hatziagelaki E, Pergialiotis V, et al. Hyperandrogenemia in women with polycystic ovary syndrome: prevalence, characteristics and association with body mass index. Horm Mol Biol Clin Investig. 2017;29(3):105-11.

11. Gaddas M, Chaouache N, Ajina M, et al. Does hyperandrogenic statute in the polycystic ovary syndrome constitutes an obstacle to the success of in vitro fertilization? J Gynecol Obstet Biol Reprod. 2016;45(9):1091-8.

12. Yang W, Yang $R$, Yang $S$, et al. Infertile polycystic ovary syndrome patients undergoing in vitro fertilization with the gonadotropin-releasing hormoneantagonist protocol: role of hyperandrogenism. Gynecol Endocrinol. 2018 Aug;34(8):715-8.

13. Ramezanali $F$, Ashrafi M, Hemat M, et al. Assisted reproductive outcomes in women with different polycystic ovary syndrome phenotypes: the predictive value of anti-Mullerian hormone. Reprod BioMed Online. 2016; 32(5):503-12.

14. Rotterdam ESHRE/ASRM-Sponsored PCOS consensus workshop group. Revised 2003 consensus on diagnostic criteria and long-term health risks related to polycystic ovary syndrome (PCOS). Hum Reprod. 2004;19(1):41-7.

15. Brassard M, AinMelk Y, Baillargeon JP. Basic infertility including polycystic ovary syndrome. Med Clin North Am. 2008;92(5):1163-92.

16. Leary C, Leese HG, Sturmey RG. Human embryos from overweight and obese women display phenotypic and metabolic abnormalities. Hum Reprod. 2015;30(1):122-32.

17. Valckx SD, De Pauw I, De Neubourg D, et al. BMI-related metabolic composition of the follicular fluid of women undergoing assisted reproductive treatment and the consequences for oocyte and embryo quality. Hum Reprod. 2012;27(12):3531-9.

18. Kimura $\mathrm{S}$, Matsumoto T, Matsuyama R, et al. Androgen receptor function in folliculogenesis and its clinical implication in premature ovarian failure. Trends Endocrinol Metab. 2007;18(5):183-9.

19. Nekoonam S, Naji M, Nashtaei MS, et al. Expression of AKT1 along with AKT2 in granulosa-lutein cells of hyperandrogenic PCOS patients. Arch Gynecol Obstet. 2017;295:1041-50.

20. Freis A, Renke $\mathrm{T}$, Kammerer U, et al. Effects of a hyperandrogenaemic state on the proliferation and decidualization potential in human endometria stromal cells. Arch Gynecol Obstet. 2017;295:1005-13.

21. Cermik D, Selam B, Taylor HS. Regulation of HOXA-10 expression by testosterone in vitro and in the endometrium of patients with polycystic ovary syndrome. J Clin Endocrinol Metab. 2003;88:238-43.

Ready to submit your research? Choose BMC and benefit from:

- fast, convenient online submission

- thorough peer review by experienced researchers in your field

- rapid publication on acceptance

- support for research data, including large and complex data types

- gold Open Access which fosters wider collaboration and increased citations

- maximum visibility for your research: over $100 \mathrm{M}$ website views per year

At $\mathrm{BMC}$, research is always in progress.

Learn more biomedcentral.com/submissions 